Gut, 1968, 9, 425-437

\title{
Absorption and malabsorption of glycine and glycine peptides in man
}

\author{
I. L. CRAFT, D. GEDDES, C. W. HYDE, IRENE J. WISE, AND \\ D. M. MATTHEWS \\ From the Professorial Surgical Unit and the Department of Chemical Pathology, \\ Westminster Medical School, London
}

The 'glycine tolerance test', an oral dose of glycine followed by analysis of peripheral blood samples, was first used for investigating amino acid absorption more than 30 years ago by Heath and Fullerton (1935), who, though they did not investigate any cases of unequivocal malabsorption, concluded that the test gave 'no useful information regarding the rate of absorption from the gastrointestinal tract'. Though the test has been used sporadically ever since in the investigation of gastrointestinal disease, particularly tropical sprue, pancreatic insufficiency, and intestinal resections (Erf and Rhoads, 1940; West, Wilson, and Eyles, 1946; Drew, Dixon, and Samuel, 1947; Anfanger and Heavenrich, 1949; Althausen, Doig, Uyeyama, and Weiden, 1950; Saint and Weiden, 1952; Butterworth, Santini, and Perez-Santiago, 1958), it has never become generally popular, nor has the similar methionine tolerance test (Harper and Uyeyama, 1948; Althausen, Uyeyama, and Simpson, 1949). The published normal values for both these tests are based on few observations, and in several cases these are quite inadequately reported, only mean values being given. Even Butterworth et al, in their investigation of the glycine test in tropical sprue, which is the best account of it, report only nine normal curves. A major disadvantage of the test as usually carried out has been methodological: the colorimetric estimation of amino-acid nitrogen (Folin, 1922) is relatively non-specific, while both the standard gasometric estimations of alpha-amino nitrogen (Hamilton and Van Slyke, 1943), and specific estimation of glycine (Alexander, Landwehr, and Seligman, 1945) are technically fairly difficult, time-consuming, and unsuited to the estimation of numerous samples.

The advent of rapid colorimetric ninhydrin methods for plasma alpha-amino nitrogen $\left(\alpha-\mathrm{NH}_{2} \mathrm{~N}\right)$, which are nearly as specific as the sasometric determination, and of a more convenient method for specific glycine estimation, combined with recent advances in our knowledge of the physiology of absorption of protein digestion products (Crane, 1961; Matthews and Laster, 1965a), which have radically altered older views, suggested a reinvestigation of the glycine tolerance test. The object of the present work was to do this, to investigate the absorption of glycylglycine (diglycine) and glycylglycylglycine (triglycine) under similar conditions, and to apply tests of free and peptide glycine absorption in various gastrointestinal disorders.

The investigation of peptide absorption in man seemed of particular interest (1) because recent investigations in animals have suggested that small peptides are not hydrolysed mainly in the intestinal lumen, as previously supposed, but transported into the cells of the intestinal mucosa and hydrolysed within them, subsequently being transported onwards and entering the blood as free amino acids (eg, Newey and Smyth, 1962; Smyth, 1964), and (2) because of suggestions that intestinal peptidases might be deficient in idiopathic steatorrhoea. Such deficiency might be either $(a)$ primary deficiency of one particular (hypothetical) peptidase, allowing accumulation of some toxic breakdown product of gliadin and implicated in the pathogenesis of gluten-induced enteropathy (Frazer, 1960; Frazer, Schneider, Morgan, Sammons, and Hayward, 1964; Pittman and Pollitt, 1966; Kowlessar, 1967) or (b) secondary, and probably general, deficiency of peptidases due to reduction in number of absorptive cells or extensive cellular disorganization (Crane and Neuberger, 1960a, b). We were interested particularly in the second possibility, since general peptidase deficiency might be revealed by the use of a single readily available peptide such as diglycine, and if such deficiency existed, malabsorption of the peptide might be more marked than that of the free amino acid. In this case, tests of peptide absorption might be more sensitive in diagnostic use than those of absorption of free amino acids.

Of the several peptidases which have been demonstrated in intestinal mucosa (Smith, E. L., 1960; Messer, Anderson, and Townley, 1961; 
Robinson, 1963), only three are likely to be concerned in the hydrolysis of diglycine and triglycine. Glycylglycine dipeptidase, a highly specific enzyme, hydrolyses diglycine, and aminotripeptidase, specific for tripeptides as a group, hydrolyses triglycine. Leucine aminopeptidase, an enzyme with a broad range of activity, may play some part in hydrolysis of diglycine and triglycine, though its activity towards them is relatively low.

\section{METHODS}

Tolerance tests were carried out in resting subjects after an overnight fast. After taking an initial venous blood sample, the dose of amino acid or peptide was given in $250 \mathrm{ml}$ water flavoured with sugar-free fruit squash, followed by a second $250 \mathrm{ml}$ of flavoured water. Heparinized blood samples were taken at $15,30,45,60,90,120$, 180 , and 240 minutes after the oral dose. Plasma was separated within 30 minutes for $\alpha-\mathrm{NH}_{2} \mathrm{~N}$ and glycine determination and chromatography. The dose of glycine was $10 \mathrm{~g}$ per $70 \mathrm{~kg}$, and adapted in proportion to body weight. The doses of diglycine and triglycine were calculated to contain the same amount of nitrogen (or number of amino acid units) as the dose of free glycine, ie, $8.8 \mathrm{~g}$ per $70 \mathrm{~kg}$ of diglycine and $8.4 \mathrm{~g}$ per $70 \mathrm{~kg}$ of triglycine.

PLASMA $\alpha-\mathrm{NH}_{2} \mathrm{~N}$ This was estimated by the colorimetric ninhydrin method (Matthews, Muir, and Baron, 1964). It is a measure of total amino acid concentration. Plasma glycine was estimated by a modification (Schwartz, Robertson, and Homes, 1955) of the method of Alexander et al, 1945) and expressed as glycine nitrogen; estimations were carried out in duplicate. The method is specific for free glycine, giving no colour yield with either of the two peptides.

QUALITATIVE CHROMATOGRAPHY Chromatography of plasma amino acids and peptides was carried out as follows. After deproteinization with 4 volumes of absolute ethanol at room temperature and standing overnight, the samples were centrifuged and $10 \mu$ l of supernatant was applied to the chromatogram. Ascending chromatograms were run on thin-layer cellulose (MN-polygram
Cel 300, Camlab) ${ }^{1}$, the solvent being methylethylketone: pyridine: ethanol: acetic acid: aqueous ammonium chloride (50:10:10:2:20). The ammonium chloride contained 12.5 m-equiv $\mathrm{HCl}$ and 17.5 m-equiv $\mathrm{NH}_{4} \mathrm{OH}$ made up to $100 \mathrm{ml}$ with water. Improved separation was obtained by a double run. For two-dimensional chromatograms, the second solvent was methanol:pyridine:water (60:60:60) (Smith, I., 1960). Detection was by spraying with $0.5 \%$ ninhydrin in $n$-butanol, followed by drying at room temperature and heating briefly in steam.

The significance of differences in means was estimated by the t test.

\section{RESULTS}

NORMAL SUBJECTS The normal control subjects were medical students and medical and technical staff. In many cases, both glycine and diglycine tests were carried out in the same individual. Plasma $\alpha-\mathrm{NH}_{2} \mathrm{~N}$ was determined in all cases, and specific glycine estimations also carried out in some subjects.

Changes in plasma $\alpha-\mathrm{NH}_{2} \mathrm{~N}$ after glycine, diglycine, and triglycine The changes in plasma $\alpha-\mathrm{NH}_{2} \mathrm{~N}$ after glycine (20 subjects), diglycine (15 subjects), and triglycine (two subjects) are shown in Figs. 1 and 2 , and the increases in concentration over the fasting level, after glycine and diglycine, summarized in Table I. After glycine, the $\alpha-\mathrm{NH}_{2} \mathbf{N}$ concentration rises to a peak at 30 to 60 minutes, nine $(45 \%)$ of the subjects having the peak at 45 minutes. The peak is followed by a fall to normal values at three to four hours. After diglycine, there is a steeper rise to a higher and earlier peak at 30 to 45 minutes, seven $(48 \%)$ of the subjects having the peak at 30 minutes. At 30 minutes, the mean increase in $\alpha-\mathrm{NH}_{2} \mathrm{~N}$ concentration after diglycine $(5.08 \mathrm{mg}$ per $100 \mathrm{ml}$, SE 0.33) is significantly higher than that following glycine (3.46 mg per $100 \mathrm{ml}$, SE 0.20) $\mathrm{P}<0.001)$. In the two triglycine tests, very high peak concentrations were attained at 30 minutes $(14.4$ and $12.7 \mathrm{mg}$ per $100 \mathrm{ml})$, the mean peak value $(13.5 \mathrm{mg}$ per $100 \mathrm{ml})$ ${ }^{1}$ Glass Limited.

\section{TABLE I}

INCREASES IN PLASMA $\alpha-\mathrm{NH}_{2} \mathrm{~N}$ AND GLYCINE N FOLLOWING GLYCINE AND DIGLYCINE

\begin{tabular}{|c|c|c|c|c|c|c|c|c|}
\hline \multirow[t]{3}{*}{ Time (min) } & \multicolumn{4}{|l|}{ After Glycine } & \multicolumn{4}{|l|}{ After Diglycine } \\
\hline & \multicolumn{2}{|c|}{$a-\mathrm{NH}_{2} \mathrm{~N}(\mathrm{mg} / 100 \mathrm{ml}) n=20$} & \multicolumn{2}{|c|}{ Glycine $N(\mathrm{mg} / 100 \mathrm{ml}) n=7$} & \multicolumn{2}{|c|}{$a-N_{2} N(m g / 100 m l) n=15$} & \multicolumn{2}{|c|}{ Glycine $N(\mathrm{mg} / 100 \mathrm{ml}) n=7$} \\
\hline & Mean & Range & Mean & Range & Mean & Range & Mean & Range \\
\hline 15 & $1 \cdot 32 \pm 0.21^{1}$ & $0-3 \cdot 5$ & 0.95 & $0 \cdot 3-1 \cdot 6$ & $1.98 \pm 0.32$ & $0-3 \cdot 4$ & 1.80 & $1 \cdot 1-2 \cdot 5$ \\
\hline 30 & $3 \cdot 46 \pm 0 \cdot 20$ & $1 \cdot 7-5 \cdot 3$ & $2 \cdot 26$ & $1 \cdot 6-3 \cdot 3$ & $5.08 \pm 0.33$ & $3 \cdot 5-8 \cdot 1$ & $4 \cdot 40$ & $3 \cdot 4-5 \cdot 5$ \\
\hline 45 & $4 \cdot 18 \pm 0 \cdot 22$ & $2 \cdot 8-6 \cdot 3$ & $3 \cdot 19$ & $2 \cdot 3-4 \cdot 4$ & $4.62 \pm 0.35$ & $2 \cdot 8-6 \cdot 6$ & $3 \cdot 80$ & $2 \cdot 3-5 \cdot 7$ \\
\hline 60 & $3.81 \pm 0.26$ & $2 \cdot 2-7 \cdot 2$ & $2 \cdot 80$ & $1 \cdot 9-4 \cdot 4$ & $3.28 \pm 0.27$ & $1 \cdot 6-5 \cdot 2$ & $2 \cdot 70$ & $1 \cdot 8-4 \cdot 0$ \\
\hline 90 & $1.67 \pm 0.21$ & $0 \cdot 3-4 \cdot 0$ & $1 \cdot 78$ & $0.8-2 \cdot 9$ & $1.76 \pm 0.23$ & $0 \cdot 5-3 \cdot 4$ & 1.48 & $1 \cdot 1-1 \cdot 7$ \\
\hline 120 & $0.82 \pm 0.14$ & $0-2 \cdot 0$ & 1.04 & $0 \cdot 6-1 \cdot 7$ & $0.81 \pm 0.17$ & $-0 \cdot 6-2 \cdot 0$ & 0.97 & $0 \cdot 6-1 \cdot 3$ \\
\hline 180 & $0.06 \pm 0.11$ & $-0.5-1 \cdot 4$ & 0.50 & $0 \cdot 3-1 \cdot 0$ & $0.15 \pm 0.12$ & $-0 \cdot 7-0.9$ & 0.56 & $0 \cdot 4-0 \cdot 7$ \\
\hline 240 & $-0.09 \pm 0.10$ & $-0 \cdot 8-0 \cdot 8$ & 0.48 & $0 \cdot 2-0 \cdot 7$ & $-0.18 \pm 0.08$ & $-0.7-0.3$ & 0.40 & $0 \cdot 3-0 \cdot 7$ \\
\hline
\end{tabular}

${ }^{1}$ Standard error of mean 


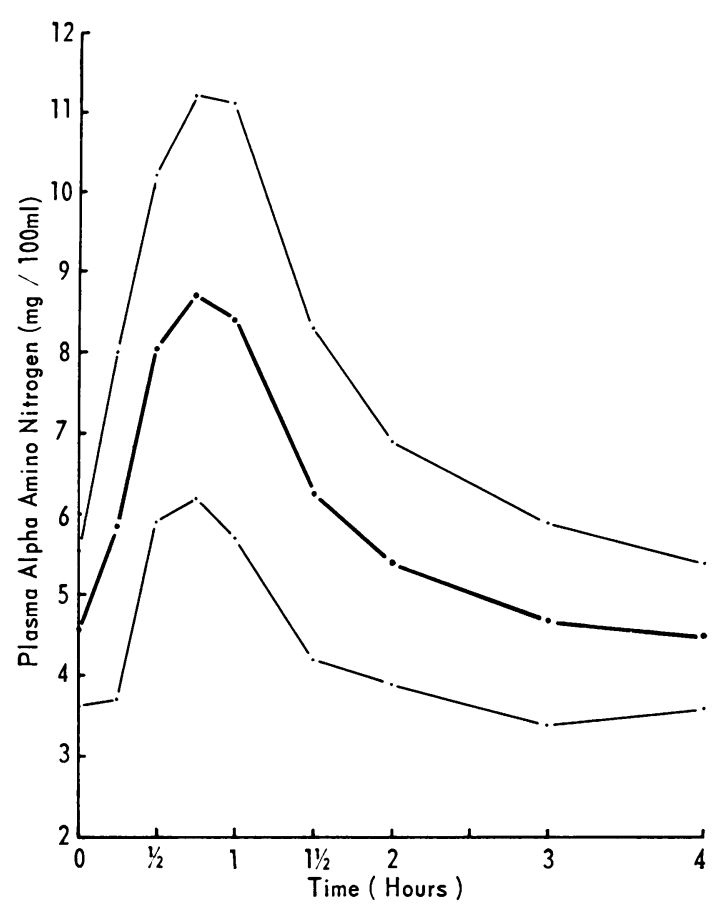

FIG. 1a

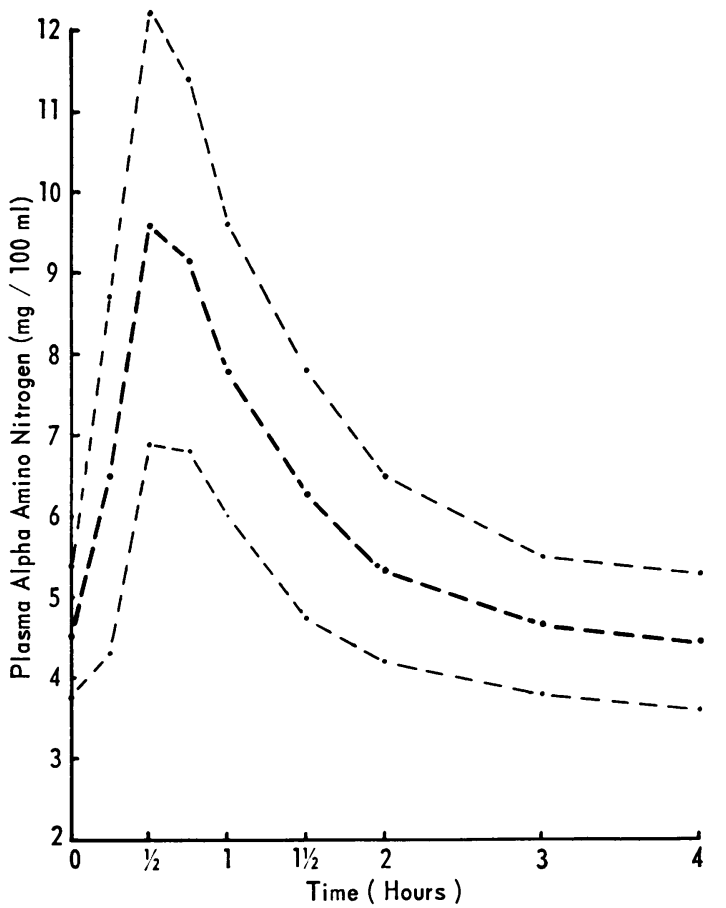

FIG. $1 \mathrm{~b}$

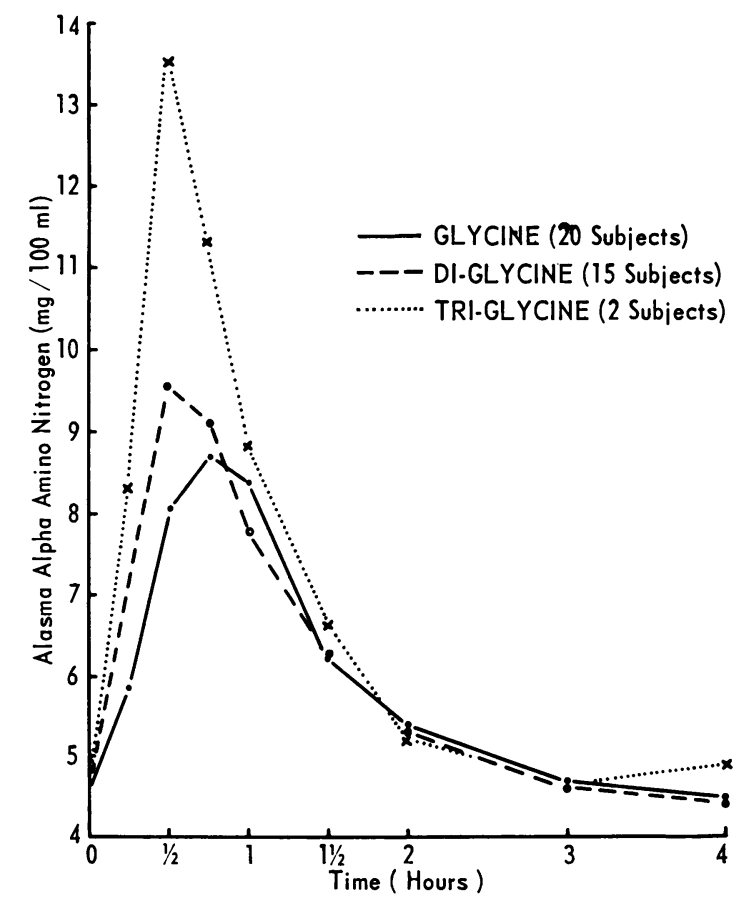

FIG. 1. Changes in plasma $\alpha-\mathrm{NH}_{2} \mathrm{~N}$. a After glycine (mean $\pm 2 S D)$

$b$ After diglycine (mean $\pm 2 S D$ )

FIG. 2. Comparison of mean changes in plasma $\alpha-\mathrm{NH}_{2} \mathrm{~N}$ after glycine, diglycine, and triglycine. - glycine; - - - diglycine; ...triglycine.

FIG. 2 
TABLE II

REPRODUCIBILITY OF GLYCINE TOLERANCE TEST IN NORMAL SUBJECTS

\begin{tabular}{|c|c|c|c|c|c|}
\hline \multirow[t]{2}{*}{ Subject } & \multicolumn{2}{|c|}{ Time of Peak (min) } & \multicolumn{3}{|c|}{ Peak Concentration of a- $\mathrm{NH}_{2} \mathrm{~N}(\mathrm{mg} / 100 \mathrm{ml})$} \\
\hline & First Test & Second Test & First Test $(A)$ & Second Test $(B)$ & $B$ as $\%$ of $A$ \\
\hline $\begin{array}{l}1 \\
2 \\
3 \\
4 \\
5 \\
6\end{array}$ & $\begin{array}{l}45 \\
45 \\
60 \\
45 \\
30 \\
30\end{array}$ & $\begin{array}{l}30 \\
45 \\
60 \\
60 \\
45 \\
45\end{array}$ & $\begin{array}{r}9.1 \\
7.6 \\
10.9 \\
11.2 \\
9.6 \\
8.6\end{array}$ & $\begin{array}{r}8.7 \\
9.9 \\
7.1 \\
12.6 \\
9.2 \\
6.7\end{array}$ & $\begin{array}{r}96 \\
130 \\
65 \\
112 \\
96 \\
78\end{array}$ \\
\hline & & & $9 \cdot 5$ & 9.0 & 96 Mean \\
\hline
\end{tabular}

falling more than 2 SD from the peak value for diglycine. In two individuals, all three tests were performed, and in both of these, triglycine gave the highest peaks and glycine the lowest, diglycine peaks being intermediate.

In six subjects, the glycine tolerance test was repeated after an interval of several days to several weeks. Table II indicates the reproducibility of the results. The peak value for $\alpha-\mathrm{NH}_{2} \mathrm{~N}$ in the second test varied from $65 \%$ to $130 \%$ of the peak value in the first test, and the times of peak values varied by not more than 15 minutes. There was no suggestion of any systematic difference between the first and second tests, and the mean peak value in the second test was approximately the same as that in the first.

Changes in plasma glycine nitrogen after glycine, diglycine, and triglycine The results obtained by specific determination of glycine (expressed as glycine nitrogen) following glycine, diglycine, and triglycine (Fig. 3) show a similar pattern to those obtained by determination of $\alpha-\mathrm{NH}_{2} \mathrm{~N}$, the rise after the peptide being steeper and attaining a higher and earlier peak than after the free amino acid, and the highest mean peak being obtained after the tripeptide. At 30 minutes, the mean increase in glycine nitrogen concentration is greater after diglycine $(4.40 \mathrm{mg}$ per $100 \mathrm{ml}$, SE $0 \cdot 24)$ than after glycine $(2.26 \mathrm{mg}$ per $100 \mathrm{ml}, \mathrm{SE} 0.24)(\mathrm{P}<0.001)$. These curves also suggest a tendency to a more rapid return towards normal values after the peptides than after the amino acid. Increases in concentration over the fasting value are summarized in Table $I$.

Figure 4 compares the mean increases in $\alpha-\mathrm{NH}_{2} \mathrm{~N}$ and in glycine nitrogen after glycine and diglycine respectively. The curves for $\alpha-\mathrm{NH}_{2} \mathrm{~N}$ (representing total amino acids), though substantially higher in the earlier part of the test than those for glycine, are reasonably similar in shape to glycine nitrogen curves, and the same general similarity was seen in individual results.

Changes in other plasma amino acids after glycine, diglycine, and triglycine Chromatography of samples from glycine tolerance tests showed a large increase in glycine, corresponding with the quantitative results. There was also a moderate increase in alanine, peaking at approximately the same time as the glycine peak, and in some cases, slight increases in serine, glutamic acid, and aspartic acid were noted. The results in diglycine and triglycine tests were similar. The increase in glycine was confined to the free amino acid; no trace of diglycine or triglycine was detected.

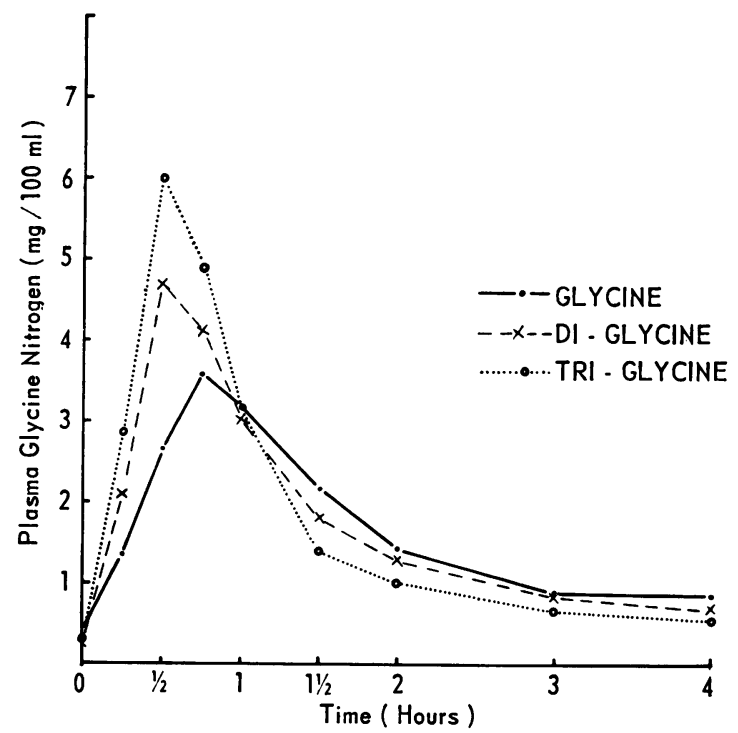

FIG. 3. Changes in plasma glycine nitrogen after glycine, diglycine, and triglycine.

PATIENTS WITH NON-FUNCTIONAL OR BYPASSED PYLORUS This group included patients (Table III) with partial gastrectomy (nos. 3, 20, 28, and 29), total gastrectomy (no. 30), vagotomy and pyloroplasty (nos. 2, 12, and 15), pyloroplasty alone (no. 5), and vagotomy and gastroenterostomy (nos. 8 and 9). Eight of the total of 11 patients (nos. 2, 5, 8, 9, $12,28,29$, and 30 ) had both glycine and diglycine tolerance curves, and in some cases specific glycine 


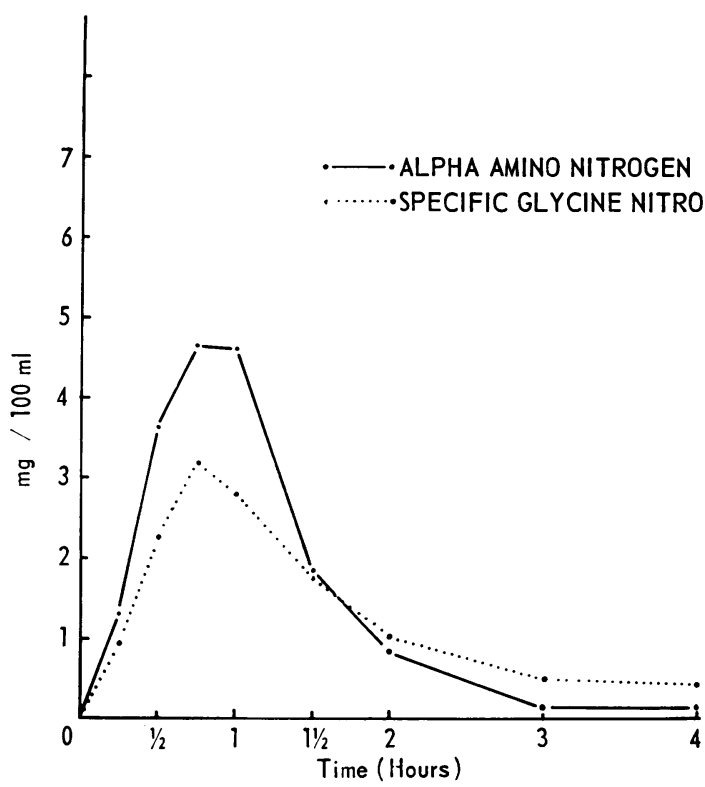

FIG. $4 \mathrm{a}$

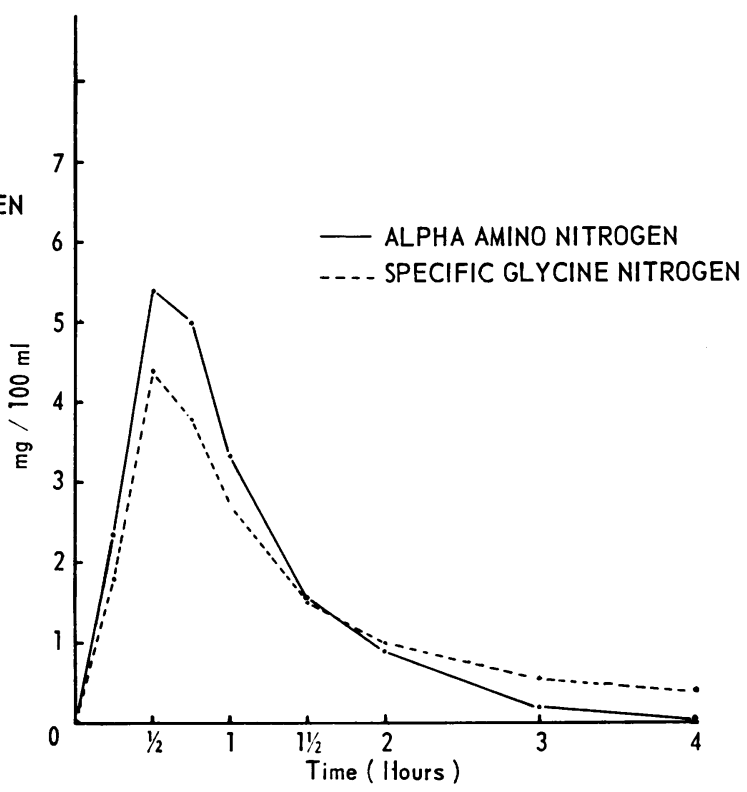

FIG. $4 b$

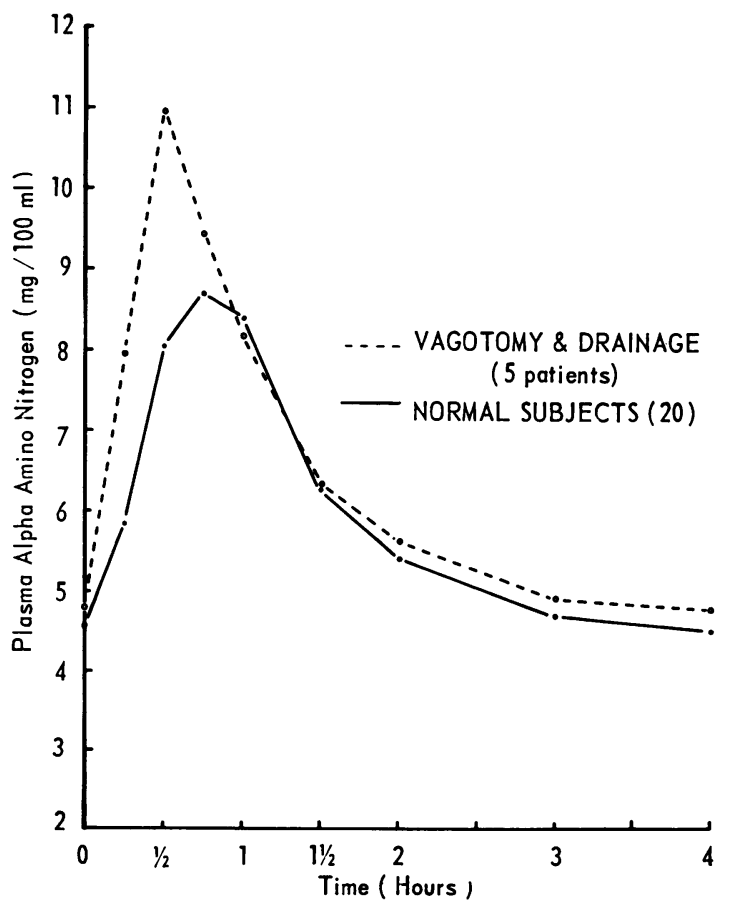

FIG. 4. Comparison of increases in $\alpha-\mathrm{NH}_{2} \mathrm{~N}$ and glycine $\mathrm{N}$ after (a) glycine and (b) diglycine.

- — $\cdot \alpha$-amino nitrogen:

- - - specific glycine nitrogen.

FIG. 5. Mean changes in plasma $\alpha-\mathrm{NH}_{2} \mathrm{~N}$ after glycine in normal subjects and in five patients with abnormally high-peaking curves.

- - vagotomy and drainage

(5 patients); - normals $(20)$.

FIG. 5 
TABLE III

INCREASES IN PLASMA $\alpha$-NH 2 N AND GLYCINE N IN PATIENTS WITH ABSENT OR NON-FUNCTIONAL PYLORUS, AFTER GLYCINE AND DIGLYCINE, DURING FIRST HOUR OF TEST

\begin{tabular}{|c|c|c|c|c|c|c|c|c|c|c|}
\hline \multirow{3}{*}{$\begin{array}{l}\text { Patient } \\
\text { No. }\end{array}$} & \multirow[t]{3}{*}{ Condition } & \multirow[t]{3}{*}{ Age } & \multirow[t]{3}{*}{$\operatorname{Sex}$} & \multirow{3}{*}{$\begin{array}{l}\text { Weight } \\
(k g)\end{array}$} & \multirow{3}{*}{$\begin{array}{l}\text { Time } \\
(\min )\end{array}$} & \multicolumn{5}{|c|}{ Increase in $\alpha-\mathrm{NH}_{2} \mathrm{~N}$ and Glycine $\mathrm{N}$} \\
\hline & & & & & & \multicolumn{2}{|c|}{ Glycine Test } & \multicolumn{2}{|c|}{ Diglycine Test } & \multirow[t]{2}{*}{ Remarks } \\
\hline & & & & & & $\begin{array}{l}a-\mathrm{NH}_{2} \mathrm{~N} \\
(\mathrm{mg} / 100 \\
\mathrm{ml})\end{array}$ & $\begin{array}{l}\text { Glycine } N \\
(\mathrm{mg} / 100 \\
\mathrm{ml})\end{array}$ & $\begin{array}{l}a-\mathrm{NH}_{2} \mathrm{~N} \\
(\mathrm{mg} / 100 \\
\mathrm{ml})\end{array}$ & $\begin{array}{l}\text { Glycine N } \\
(\mathrm{mg} / 100 \\
\mathrm{ml})\end{array}$ & \\
\hline 3 & Partial gastrectomy & 50 & $\mathbf{M}$ & 42 & $\begin{array}{l}15 \\
30 \\
45 \\
60\end{array}$ & $\begin{array}{l}7 \cdot 4 \\
6 \cdot 1 \\
4 \cdot 0 \\
2 \cdot 3 \mathrm{H}^{1}\end{array}$ & $\begin{array}{l}4 \cdot 2 \\
3.0 \\
2 \cdot 3 \\
1 \cdot 9 \mathrm{H}\end{array}$ & - & - & \\
\hline 20 & Partial gastrectomy & 63 & $\mathbf{M}$ & 57 & $\begin{array}{l}15 \\
30 \\
45 \\
60\end{array}$ & $\begin{array}{l}0.9 \\
2.2 \\
3.5 \\
2.5\end{array}$ & - & - & - & Faecal fat $10 \mathrm{~g} / 24$ hours \\
\hline 28 & Partial gastrectomy & 53 & $\mathbf{F}$ & 67 & $\begin{array}{l}15 \\
30 \\
45 \\
60\end{array}$ & $\begin{array}{l}3 \cdot 3 \\
5 \cdot 0 \\
4 \cdot 2 \\
2 \cdot 4\end{array}$ & $\begin{array}{l}2.0 \\
3.9 \\
3.4 \\
2.6 \mathrm{H}\end{array}$ & $\begin{array}{l}3 \cdot 3 \\
6 \cdot 8 \\
5 \cdot 4 \\
4 \cdot 2\end{array}$ & $\begin{array}{l}3 \cdot 0 \\
5 \cdot 4 \\
5 \cdot 1 \\
3 \cdot 7\end{array}$ & \\
\hline 29 & Partial gastrectomy & 77 & $\mathbf{F}$ & 67 & $\begin{array}{l}15 \\
30 \\
45 \\
60\end{array}$ & $\begin{array}{l}3.4 \\
5.0 \\
5.0 \\
3.7\end{array}$ & $\begin{array}{l}3.5 \\
5.8 \\
4.8 \\
3.8 \mathrm{H}\end{array}$ & $\begin{array}{l}7 \cdot 6 \\
5 \cdot 3 \\
4 \cdot 7 \\
2 \cdot 6 \mathrm{H}\end{array}$ & $\begin{array}{l}5 \cdot 4 \\
6 \cdot 3 \\
5 \cdot 0 \\
3 \cdot 9 \mathrm{H}\end{array}$ & \\
\hline 30 & Total gastrectomy & 49 & $\mathbf{M}$ & 70 & $\begin{array}{l}15 \\
30 \\
45 \\
60\end{array}$ & $\begin{array}{l}3 \cdot 5 \\
3.4 \\
4 \cdot 8 \\
3 \cdot 8\end{array}$ & $\begin{array}{l}2.9 \\
2 \cdot 8 \\
3 \cdot 5 \\
-\mathrm{H}\end{array}$ & $\begin{array}{l}4.0 \\
3.8 \\
3.3 \\
1.5\end{array}$ & $\begin{array}{l}3.7 \\
4.0 \\
2.5 \\
1.6 \mathrm{H}\end{array}$ & \\
\hline 12 & Vagotomy and pyloroplasty & 53 & $\mathbf{F}$ & 73 & $\begin{array}{l}15 \\
30 \\
45 \\
60\end{array}$ & $\begin{array}{l}3 \cdot 3 \\
7 \cdot 1 \\
5 \cdot 0 \\
3 \cdot 9 \mathrm{H}\end{array}$ & - & $\begin{array}{l}6 \cdot 4 \\
6 \cdot 7 \\
4 \cdot 4 \\
3 \cdot 2 \mathrm{H}\end{array}$ & - & \\
\hline 15 & Vagotomy and pyloroplasty & 32 & $\mathbf{M}$ & 64 & $\begin{array}{l}15 \\
30 \\
45 \\
60\end{array}$ & $\begin{array}{l}5 \cdot 2 \\
6 \cdot 7 \\
5 \cdot 3 \\
3 \cdot 8 \mathrm{H}\end{array}$ & - & - & - & \\
\hline 2 & Vagotomy and pyloroplasty & 37 & $\mathbf{M}$ & 60 & $\begin{array}{l}15 \\
30 \\
45 \\
60\end{array}$ & $\begin{array}{l}1.9 \\
4.3 \\
3.2 \\
1.4\end{array}$ & $\begin{array}{l}1.9 \\
3.7 \\
2.6 \\
1.7 \mathrm{H}\end{array}$ & $\begin{array}{l}6.7 \\
5.0 \\
2.9 \\
1.7 \mathrm{H}\end{array}$ & $\begin{array}{l}6 \cdot 0 \\
4 \cdot 0 \\
2 \cdot 3 \\
1 \cdot 5 \mathrm{H}\end{array}$ & Faecal fat $7 \cdot 2 \mathrm{~g} / 24$ hours \\
\hline 5 & Pyloroplasty & 52 & $\mathbf{F}$ & 54 & $\begin{array}{l}15 \\
30 \\
45 \\
60\end{array}$ & $\begin{array}{l}4.4 \\
7 \cdot 2 \\
5 \cdot 1 \\
3 \cdot 8 \mathrm{H}\end{array}$ & $\begin{array}{l}3.7 \\
4.9 \\
4 \cdot 0 \\
3.4 \mathrm{H}\end{array}$ & $\begin{array}{l}7 \cdot 2 \\
7 \cdot 2 \\
5 \cdot 3 \\
3 \cdot 6 \mathrm{H}\end{array}$ & $\begin{array}{l}5.5 \\
4.7 \\
4.2 \\
3.0 \mathrm{H}\end{array}$ & $\begin{array}{l}\text { Patient had biliary } \\
\text { cirrhosis. Faecal fat } \\
30 \mathrm{~g} / 24 \text { hours }\end{array}$ \\
\hline 8 & $\begin{array}{l}\text { Vagotomy and gastro- } \\
\text { enterostomy }\end{array}$ & 46 & $\mathbf{M}$ & 80 & $\begin{array}{l}15 \\
30 \\
45 \\
60\end{array}$ & $\begin{array}{l}3.7 \\
8 \cdot 1 \\
5 \cdot 3 \\
3 \cdot 7 \mathrm{H}\end{array}$ & - & $\begin{array}{l}4 \cdot 9 \\
5 \cdot 5 \\
3 \cdot 1 \\
2 \cdot 0 \mathrm{H}\end{array}$ & - & \\
\hline 9 & $\begin{array}{l}\text { Vagotomy and gastro- } \\
\text { enterostomy }\end{array}$ & 35 & $\mathbf{M}$ & 80 & $\begin{array}{l}15 \\
30 \\
45 \\
60\end{array}$ & $\begin{array}{l}2 \cdot 4 \\
4 \cdot 7 \\
4 \cdot 3 \\
3 \cdot 9\end{array}$ & - & $\begin{array}{l}5 \cdot 1 \\
8 \cdot 2 \\
4 \cdot 6 \\
3 \cdot 1 \mathrm{H}\end{array}$ & - & \\
\hline
\end{tabular}

${ }^{1} \mathrm{H}$ The increase exceeds that found in normals

after giving this substance, and in three cases, peaks occurred at 15 minutes. Peaks as early as 15 minutes did not occur in normal subjects. In some cases (nos. 2, 9, and 29), the increase in $\alpha-\mathrm{NH}_{2} \mathrm{~N}$ after diglycine was excessive while that after glycine was within normal limits. The tendency to curves with high and early peaks was confirmed in those patients in whom glycine was estimated specifically.

In eight patients given both tests, the mean 15-minute increase in $\alpha-\mathrm{NH}_{2} \mathrm{~N}$ was higher after diglycine $(5.6 \mathrm{mg}$ per $100 \mathrm{ml}$, SE 0.59$)$ than after diglycine (nos. 2, 5, 8, 9, 12, and 29), the increase in $\alpha-\mathrm{NH}_{2} \mathrm{~N}$ exceeded the increase found in normals 
TABLE IV

INCREASES IN PLASMA $\alpha-\mathrm{NH}_{2} \mathrm{~N}$ AFTER GLYCINE AND DIGLYCINE, IN PATIENTS WITH INTESTINAL DISORDERS AND PANCREATIC STEATORRHOEA ${ }^{1}$

\begin{tabular}{|c|c|c|c|c|c|c|c|c|c|c|c|}
\hline \multirow{2}{*}{$\begin{array}{l}\text { Patient } \\
\text { No. }\end{array}$} & \multirow[t]{2}{*}{ Condition } & \multirow[t]{2}{*}{ Age } & \multirow[t]{2}{*}{ Sex } & \multirow{2}{*}{$\begin{array}{l}\text { Weight } \\
(k g)\end{array}$} & \multicolumn{2}{|c|}{ Glycine Test } & \multicolumn{2}{|c|}{ Diglycine Test } & \multirow{2}{*}{$\begin{array}{l}\text { Faecal } \\
\text { Fat } \\
\text { (g/ } \\
\text { N } 24 \text { hours) }\end{array}$} & \multirow{2}{*}{$\begin{array}{l}\text { Urine } \\
\text { Xylose } \\
(g / \\
\text { s) } 5 \text { hours) }\end{array}$} & \multirow[t]{2}{*}{ Remarks } \\
\hline & & & & & $\begin{array}{rr}\text { Time } & I \\
(\min ) & i \\
& 1 \\
& 1\end{array}$ & $\begin{array}{l}\text { Increase } \\
\text { in } a-N H_{2} N \\
(m g / \\
100 \mathrm{ml})\end{array}$ & $\begin{array}{c}\text { Time } \\
V(\min )\end{array}$ & $\begin{array}{l}\text { Increase } \\
\text { in a- } \mathrm{NH}_{2} \mathrm{~N} \\
(\mathrm{mgl} \\
100 \mathrm{ml})\end{array}$ & & & \\
\hline 13 & $\begin{array}{l}\text { Idiopathic } \\
\text { steatorrhoea }\end{array}$ & 62 & $\mathbf{F}$ & 38 & $\begin{array}{l}15 \\
30 \\
45 \\
60 \\
90 \mathbf{P}^{2}\end{array}$ & $\begin{array}{l}0 \\
0 \cdot 1 \\
0 \cdot 8 \\
0 \cdot 8 \\
1 \cdot 3 \mathrm{~S}^{8}\end{array}$ & $\begin{array}{l}15 \\
30 \\
45 \\
60 \\
90 \mathrm{P}\end{array}$ & $\begin{array}{l}0 \\
0.5 \\
1.0 \\
1.3 \\
2.3 \mathrm{~S}\end{array}$ & 12 & $\begin{array}{l}0.9 \\
(25 \mathrm{~g} \text { dose }) \\
\mathrm{S}\end{array}$ & $\begin{array}{l}\text { Biopsy-total villous } \\
\text { atrophy } \\
\text { Gluten-free diet and } \\
\text { steroids, } 3 \text { weeks-some } \\
\text { improvement } \\
\text { Glycine } N \text { gave } \\
\text { similar curves. }\end{array}$ \\
\hline 23 & $\begin{array}{l}\text { Idiopathic steator- } \\
\text { rhoea (probable) }\end{array}$ & 46 & $\mathbf{M}$ & 44 & $\begin{array}{c}15 \\
30 \\
45 \\
60 \\
120 \mathrm{P}\end{array}$ & $\begin{array}{c}-0.4 \\
0.6 \\
1.0 \\
1.3 \\
1.6 \mathrm{~S}\end{array}$ & $\begin{array}{l}15 \\
30 \\
45 \\
60 \\
180 \mathrm{P}\end{array}$ & $\begin{array}{l}0 \\
0.4 \\
0.1 \\
0.7 \\
2.2 \mathrm{~S}\end{array}$ & 16 & - & $\begin{array}{l}\text { Gluten-free diet, } 2 \text { years- } \\
\text { no improvement } \\
\mathrm{Hb} 6.7 \mathrm{~g} / 100 \mathrm{ml} \text {, megalo- } \\
\text { blastic } \\
\mathbf{B}_{12} \text { and folic-deficient }\end{array}$ \\
\hline 27 & $\begin{array}{l}\text { Idiopathic steator- } \\
\text { rhoea }\end{array}$ & 35 & $\mathbf{M}$ & 48 & $\begin{array}{l}15 \\
30 \\
45 \\
60 \\
240 \mathrm{P}\end{array}$ & $\begin{array}{l}-0 \cdot 3 \\
0 \cdot 1 \\
0 \cdot 3 \\
0 \cdot 5 \\
1 \cdot 3 \mathrm{~S}\end{array}$ & $\begin{array}{l}15 \\
30 \\
45 \\
60 \\
90 P\end{array}$ & $\begin{array}{l}0.6 \\
0.3 \\
2 \cdot 1 \\
1.5 \\
2 \cdot 2 \mathrm{~S}\end{array}$ & 25 & $\begin{array}{l}1.0 \\
(5 g \text { dose }) \\
S\end{array}$ & $\begin{array}{l}\text { Untreated } \\
\text { Hb } 9 \cdot 4 \mathrm{~g} / 100 \mathrm{ml} \text {, megalo- } \\
\text { blastic } \\
\text { B }_{12} \text {-deficient }\end{array}$ \\
\hline 18 & $\begin{array}{l}\text { Idiopathic steator- } \\
\text { rhoea }\end{array}$ & 29 & $\mathbf{F}$ & 45 & $\begin{array}{l}15 \\
30 \\
45 \\
60 \mathrm{P}\end{array}$ & $\begin{array}{l}0 \cdot 5 \\
3 \cdot 3 \\
3 \cdot 4 \\
3 \cdot 8\end{array}$ & - & - & 12 & $\begin{array}{l}4 \cdot 2 \\
(25 \mathrm{~g} \text { dose })\end{array}$ & $\begin{array}{l}\text { On gluten-free diet- } \\
\text { biopsy improved }\end{array}$ \\
\hline 22 & $\begin{array}{l}\text { Idiopathic steator- } \\
\text { rhoea }\end{array}$ & 38 & $\mathbf{F}$ & 53 & $\begin{array}{l}15 \\
30 \\
45 P \\
60\end{array}$ & $\begin{array}{l}0 \cdot 9 \\
3 \cdot 5 \\
4 \cdot 6 \\
3 \cdot 4\end{array}$ & $\begin{array}{l}15 \\
30 \\
45 P \\
60\end{array}$ & $\begin{array}{l}1 \cdot 2 \\
4 \cdot 7 \\
7 \cdot 2 \\
6 \cdot 4\end{array}$ & $3 \cdot 7$ & $\begin{array}{l}4 \cdot 3 \\
(25 g \text { dose })\end{array}$ & $\begin{array}{l}\text { Intermittent gluten-free diet } \\
\text { Biopsy-total villous } \\
\text { atrophy } \\
\text { Flat G.T.T. }\end{array}$ \\
\hline 14 & $\begin{array}{l}\text { Idiopathic steator- } \\
\text { rhoea } \\
\text { (probable) }\end{array}$ & 49 & $\mathbf{M}$ & 70 & $\begin{array}{l}15 \\
30 \\
45 \\
60 \mathrm{P}\end{array}$ & $\begin{array}{l}2 \cdot 2 \\
2 \cdot 9 \\
4 \cdot 5 \\
4 \cdot 9\end{array}$ & $\begin{array}{l}15 \\
30 \\
45 \\
60 P\end{array}$ & $\begin{array}{l}0.6 \\
2 \cdot 0 \\
2 \cdot 6 \\
4 \cdot 6 \mathrm{~S}\end{array}$ & $8 \cdot 0$ & $\begin{array}{l}3.0 \\
(25 \mathrm{~g} \text { dose }) \\
\mathrm{S}\end{array}$ & $\begin{array}{l}\text { Hb } 9 \cdot 3 \mathrm{~g} / 100 \mathrm{ml} \text {, megalo- } \\
\text { blastic } \\
\text { B }_{12} \text {-deficient } \\
\text { Flat G.T.T. }\end{array}$ \\
\hline 17 & $\begin{array}{l}\text { Idiopathic steator- } \\
\text { rhoea }\end{array}$ & 21 & $\mathbf{F}$ & 42 & $\begin{array}{l}15 \\
30 \\
45 P \\
60\end{array}$ & $\begin{array}{l}0 \\
1 \cdot 2 \\
3 \cdot 8 \\
2 \cdot 6 \mathrm{~S}\end{array}$ & $\begin{array}{l}15 \\
30 \\
45 P \\
60\end{array}$ & $\begin{array}{l}0 \cdot 8 \\
5 \cdot 1 \\
5 \cdot 3 \\
4 \cdot 6\end{array}$ & $6 \cdot 5$ & $\begin{array}{l}7 \cdot 6 \\
(25 g \text { dose })\end{array}$ & $\begin{array}{l}\text { Subnormal vitamin } A \text { and } \\
\text { folic acid absorption }\end{array}$ \\
\hline 16 & $\begin{array}{l}\text { Crohn's disease } \\
\text { (lower ileum) }\end{array}$ & 38 & $\mathbf{M}$ & 57 & $\begin{array}{l}15 \\
30 \\
45 P \\
60\end{array}$ & $\begin{array}{l}0 \cdot 7 \\
2 \cdot 3 \\
2 \cdot 5 \\
2 \cdot 3\end{array}$ & - & - & - & - & $\begin{array}{l}\text { Laparotomy } 2 \text { years ago- } \\
50 \mathrm{~cm} \text { terminal ileum } \\
\text { involved } \\
\text { On steroids }\end{array}$ \\
\hline
\end{tabular}

${ }^{1}$ The Table shows increases in $a-\mathrm{NH}_{2} \mathrm{~N}$ for the first hour of the list, and also at the peak of the curve if this occurs later than 60 minutes. ${ }^{2} \mathbf{P}$ Peak of curve ${ }^{3} \mathrm{~S}$ Subnormal

glycine (3.2 $\mathrm{mg}$ per $100 \mathrm{ml} \mathrm{SE}, 0 \cdot 27)$. The difference was significant $(P<0.01>0.001)$. A similar difference was found in five patients in whom specific glycine determinations were made. In all these cases, the 15-minute increase was greater after diglycine than after glycine. The mean 15-minute increase after diglycine was $4.7 \mathrm{mg}$ per $100 \mathrm{ml}$ (SE 0.58) and that after glycine $2.8 \mathrm{mg}$ per $100 \mathrm{ml}$ (SE 0.37). The difference was significant $(P<0.02>0.01)$.

PATIENTS WITH INTESTINAL DISORDERS AND PANCREATIC STEATORRHOEA The tests were carried out in seven patients with a diagnosis of idiopathic steatorrhoea (nos. 13, 14, 17, 18, 22, 23, and 27 in Table IV). The criteria for a firm diagnosis of this condition included a characteristic intestinal biopsy. In two cases, the diagnosis appeared likely on other grounds, but attempts at biopsy were unsuccessful; these are indicated in Table IV by the word 'probable' under the diagnosis. After glycine, grossly depressed $\alpha-\mathrm{NH}_{2} \mathrm{~N}$ curves were found in three patients (nos. 13, 23, and 27 in Fig. 6) and a slightly subnormal 30-minute rise in one (no. 17), the others being within normal limits. Patients 13, 23, and 27 also had grossly depressed curves after diglycine, though in patient 13 , the peak after diglycine was substantially higher than that after glycine. One patient (no. 14) had a normal curve after glycine, but after diglycine the rise at $\mathbf{3 0}$ minutes was below the lower limit of normal and the peak value was later (one hour) than in any of the normal controls. The most severe abnormalities in the glycine and 


\section{T A B L E IV}

INCREASES IN PLASMA $\alpha-\mathrm{NH}_{2} \mathrm{~N}$ AFTER GLYCINE AND DIGLYCINE, IN PATIENTS WITH INTESTINAL DISORDERS AND PANCREATIC STEATORRHOEA

\begin{tabular}{|c|c|c|c|c|c|c|c|c|c|c|c|}
\hline \multirow{2}{*}{$\begin{array}{l}\text { Patient } \\
\text { No. }\end{array}$} & \multirow[t]{2}{*}{ Condition } & \multirow[t]{2}{*}{ Age } & \multirow[t]{2}{*}{$\operatorname{Sex}$} & \multirow{2}{*}{$\begin{array}{l}\text { Weight } \\
(\mathrm{kg})\end{array}$} & \multicolumn{2}{|c|}{ Glycine Test } & \multicolumn{2}{|c|}{ Diglycine Test } & \multirow{2}{*}{$\begin{array}{l}\text { Faecal } \\
\text { Fat } \\
(\mathrm{g} / \\
24 \text { hours })\end{array}$} & \multirow{2}{*}{$\begin{array}{l}\text { Urine } \\
\text { Xylose } \\
\text { (gl } \\
5 \text { hours) }\end{array}$} & \multirow[t]{2}{*}{ Remarks } \\
\hline & & & & & $\begin{array}{c}\text { Time } \\
(\text { min }) \\
\end{array}$ & $\begin{array}{l}\text { Increase } \\
\text { in a-NH } \mathrm{H}_{2} \mathrm{~N} \\
(\mathrm{mg} / \\
100 \mathrm{ml})\end{array}$ & $\begin{array}{c}\text { Time } \\
\text { (min) }\end{array}$ & $\begin{array}{l}\text { Increase } \\
\text { in a- } \mathrm{NH}_{2} \mathrm{~N} \\
(\mathrm{mgl} \\
100 \mathrm{ml})\end{array}$ & & & \\
\hline 32 & $\begin{array}{l}\text { Crohn's disease } \\
\text { (almost all small } \\
\text { gut involved) }\end{array}$ & 18 & $\mathbf{F}$ & 36 & $\begin{array}{l}15 \\
30 \\
45 \\
60 \\
90 \mathrm{P}\end{array}$ & $\begin{array}{l}0.5 \\
1.0 \\
1.2 \\
1.4 \\
1.6 \mathrm{~S}\end{array}$ & - & - & - & - & On steroids \\
\hline 11 & $\begin{array}{l}\text { Crohn's disease } \\
\text { (resection } 150 \mathrm{~cm} \\
\text { distal ileum) }\end{array}$ & 56 & $\mathbf{M}$ & 64 & $\begin{array}{l}15 \\
30 P \\
45 \\
60\end{array}$ & $\begin{array}{l}1 \cdot 5 \\
3 \cdot 4 \\
2 \cdot 9 \\
3 \cdot 2\end{array}$ & - & 一 & - & - & Resection 6 years ago \\
\hline 24 & $\begin{array}{l}\text { Crohn's disease } \\
\text { (resection } 100 \mathrm{~cm} \\
\text { distal ileum) }\end{array}$ & 58 & $\mathbf{F}$ & 72 & $\begin{array}{l}15 \\
30 \\
45 P \\
60\end{array}$ & $\begin{array}{l}0.5 \\
2 \cdot 4 \\
3 \cdot 8 \\
3 \cdot 0\end{array}$ & - & - & 10 & - & Resection 5 years ago \\
\hline 25 & $\begin{array}{l}\text { Crohn's disease } \\
\text { (resection } 100 \mathrm{~cm} \\
\text { distal ileum) }\end{array}$ & 58 & $\mathbf{M}$ & 52 & $\begin{array}{l}15 \\
30 P \\
45 \\
60\end{array}$ & $\begin{array}{l}2.6 \\
3.9 \\
3.8 \\
3.0\end{array}$ & - & - & - & - & $\begin{array}{l}\text { Resection } 18 \text { years ago } \\
\text { also partial gastrectomy } \\
9 \text { years ago }\end{array}$ \\
\hline 19 & $\begin{array}{l}\text { Resection distal } \\
\text { half of small gut for } \\
\text { mesenteric thrombosis }\end{array}$ & 67 & $\mathbf{F}$ & 54 & $\begin{array}{c}15 \\
30 \\
45 \\
60 \\
120 \mathrm{P}\end{array}$ & $\begin{array}{l}0.6 \\
0.1 \\
0.3 \\
0.8 \\
0.9 \mathrm{~S}\end{array}$ & - & - & - & - & $\begin{array}{l}\text { Resection } 2 \text { months ago- } \\
\text { oedema, electrolyte } \\
\text { disturbance } \\
\text { Glycine N gave similar } \\
\text { results. }\end{array}$ \\
\hline 6 & $\begin{array}{l}\text { Proximal resection of } \\
100 \mathrm{~cm}, 50 \mathrm{~cm} \text { distal } \\
\text { to D-J flexure-for } \\
\text { diverticula }\end{array}$ & 63 & $\mathbf{M}$ & 77 & $\begin{array}{l}15 \\
30 \\
45 P \\
60\end{array}$ & $\begin{array}{l}1 \cdot 7 \\
2 \cdot 8 \\
3 \cdot 4 \\
3 \cdot 1\end{array}$ & $\begin{array}{l}15 \\
30 \\
45 P \\
60\end{array}$ & $\begin{array}{l}1 \cdot 6 \\
4 \cdot 2 \\
5 \cdot 0 \\
2 \cdot 5\end{array}$ & 3.9 & $\begin{array}{l}1 \cdot 1 \\
(5 g \text { dose }) \\
S\end{array}$ & $\begin{array}{l}\text { Resection } \\
\text { G.T.T., delayed peak } \\
\text { at } 90 \mathrm{~min}\end{array}$ \\
\hline 26 & $\begin{array}{l}\text { Malignant abdominal } \\
\text { carcinoid (liver } \\
\text { metastases) }\end{array}$ & 64 & $\mathbf{F}$ & 60 & $\begin{array}{l}15 \\
30 \\
45 \\
60 \\
90 \mathrm{P}\end{array}$ & $\begin{array}{l}0 \cdot 4 \\
2 \cdot 0 \\
3 \cdot 2 \\
4 \cdot 1 \\
5 \cdot 6\end{array}$ & - & & - & $\begin{array}{l}0.9 \\
(5 g \text { dose }) \\
\text { S }\end{array}$ & $\begin{array}{l}\text { Severe diarrhoea } \\
\text { 5-HIAA } 42 \mathrm{mg} / 24 \text { hours }\end{array}$ \\
\hline 21 & $\begin{array}{l}\text { Acute ulcerative } \\
\text { colitis }\end{array}$ & 23 & $\mathbf{F}$ & 44 & $\begin{array}{l}15 \\
30 \\
45 \\
60 \mathrm{P}\end{array}$ & $\begin{array}{l}1 \cdot 9 \\
4 \cdot 2 \\
4 \cdot 2 \\
4 \cdot 5\end{array}$ & - & - & - & - & Severe diarrhoea \\
\hline 31 & Chronic pancreatitis & 65 & $\mathbf{M}$ & 68 & $\begin{array}{l}15 \\
30 \\
45 \\
60 \\
90 \mathrm{P}\end{array}$ & $\begin{array}{l}2 \cdot 2 \\
4 \cdot 5 \\
6 \cdot 9 \\
7 \cdot 2 \\
7 \cdot 3\end{array}$ & - & - & 9 & - & $\begin{array}{l}\text { G.T.T. normal } \\
\text { ? hepatic cirrhosis }\end{array}$ \\
\hline
\end{tabular}

diglycine tests occurred in those patients with the severest absorptive defects as judged by the faecal fat and xylose tests but the numbers are too small to make any firm generalizations. Specific glycine determinations were carried out after glycine and diglycine in patient 13; the results confirmed the findings with the $\alpha-\mathrm{NH}_{2} \mathrm{~N}$ estimations.

Chromatography of samples from this group of patients showed similar qualitative changes to those in normals after glycine and diglycine, though the glycine and alanine spots were less intense. No peptide was detected after the administration of diglycine.

In a patient (no. 16) with Crohn's disease of the lower ileum $(50 \mathrm{~cm}$ of terminal ileum had been found to be involved at laparotomy two years previously) the 30-minute increase in $\alpha-\mathrm{NH}_{2} \mathrm{~N}$ after glycine was at the lower limit of the normal range, though the curve was not definitely abnormal. On the other hand, in a patient known to have Crohn's disease involving almost the entire small intestine (no. 32), the curve was severely depressed. Three patients (nos. 11, 24, and 25) with resections of 100 to $150 \mathrm{~cm}$ of lower ileum for Crohn's disease done several years previously, had normal curves. Patient 19, who had a distal resection of about half of the small intestine for mesenteric thrombosis two months before the test, had a very severe depression of the curve following glycine; it is possible that the malabsorption was contributed to by oedema of the gut wall or other factors, since the patient had generalized oedema associated with low serum 


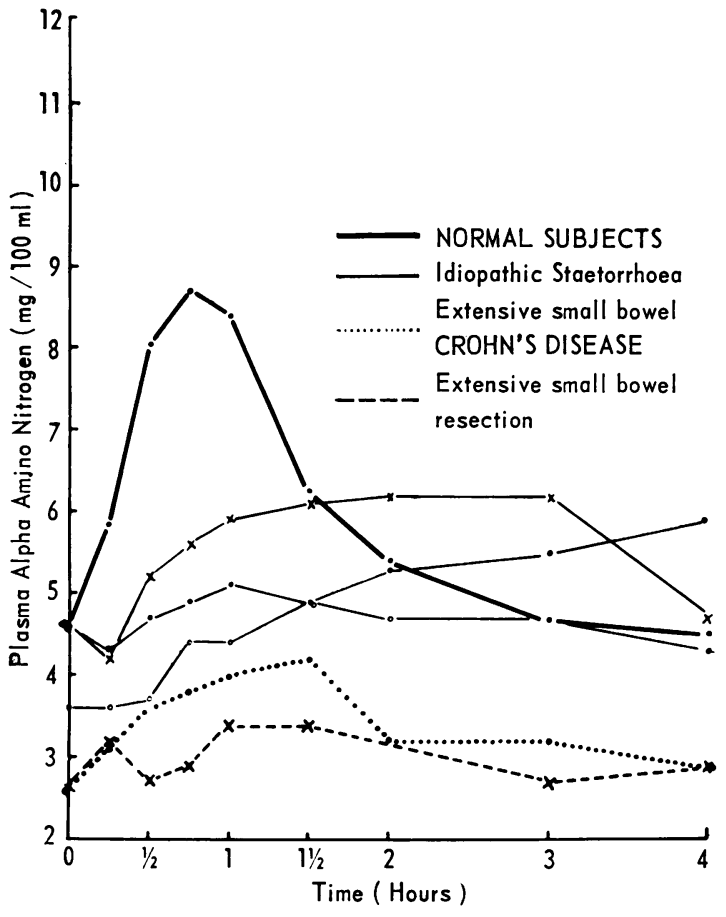

FIG. 6. Changes in plasma $\alpha-\mathrm{NH}_{2} \mathrm{~N}$ after glycine in normal subjects (mean curve) and in five patients with grossly depressed curves. - normals; - idiopathic steatorrhoea; ... extensive Crohn's disease; - - extensive small bowel resection.

proteins, and an electrolyte disturbance with hypokalaemia. Patient 6, with a proximal resection for diverticula (resection of $100 \mathrm{~cm}$ distal to the duodenojejunal flexure), had completely normal curves after glycine and diglycine, though the results of the xylose test were subnormal.

A case (no. 21) of acute ulcerative colitis had a normal curve following glycine. One of malignant carcinoid syndrome (no. 26), with severe diarrhoea and a subnormal xylose test, also had a normal curve.

A case (no. 31) of pancreatic steatorrhoea had an abnormally high curve, peaking at 90 minutes, following glycine. This patient, on laparotomy findings, was suspected of hepatic cirrhosis, though routine 'liver function tests', including transaminases, were normal.

Examples of grossly depressed glycine tolerance curves are shown in Figure 6.

\section{DISCUSSION}

We believe that the present version of the glycine tolerance test has appreciable advantages over the test as usually carried out. The use of a smaller dose of glycine than the traditional 25 or $50 \mathrm{~g}$ results in an $\alpha-\mathrm{NH}_{2} \mathrm{~N}$ curve that normally peaks not later than one hour after the dose, and is approaching fasting values in three hours. Nausea is avoided. ${ }^{1}$ It is possible that the smaller dose may prove to increase the diagnostic sensitivity of the test, though the choice of the best dose in such tests is essentially an empirical matter, and further experience will be necessary before this can be decided. Adjustment of the dose on a weight basis was used in an attempt to reduce the scatter of response. The method used to estimate $\alpha-\mathrm{NH}_{2} \mathrm{~N}$ is nearly as specific as the gasometric ninhydrin technique, and its simplicity makes it suitable for the routine laboratory. Estimation of glycine itself is relatively difficult technically, but the results suggest that alterations in plasma $\alpha-\mathrm{NH}_{2} \mathrm{~N}$ follow alterations in glycine sufficiently closely to make the former estimation adequate for diagnostic purposes. This confirms previous findings (Christensen, Cooper, Johnson, and Lynch, 1947; Butterworth et al, 1958). The fact that the increase in concentration of $\alpha-\mathrm{NH}_{2} \mathrm{~N}$ exceeds that of glycine nitrogen during the earlier part of the curve appears to be largely due to an increase in alanine. This also has been noted previously (Christensen et al, 1947). During the latter part of the curve, the plasma glycine tends to remain slightly raised, while the total $\alpha-\mathrm{NH}_{2} \mathrm{~N}$ tends to fall below the fasting value (Table I), presumably owing to depression of other amino acids. This type of phenomenon is recognized, and it is known that the effects of a large dose of one amino acid on the distribution of others are extremely complex (Hier, 1947; Christensen, Streicher, and Elbinger, 1948; Frame, 1958). The reproducibility of the test when repeated in the same individual is fairly satisfactory, and the range of response obtained in normal subjects compares very favourably with that of the standard 100-g glucose tolerance test (McDonald, Fisher, and Burnham, 1965).

PHYSIOLOGICAL CONSIDERATIONS Comparison of tolerance curves after glycine, diglycine, and triglycine suggests some interesting physiological considerations. The doses of each peptide contained the same amount of nitrogen, in other words the same number of amino acid units, as those of the free amino acid. These doses of peptide were chosen because it was expected, as the result of animal experiments (eg, Newey and Smyth, 1962), that the peptides would be rapidly hydrolysed during absorption and that the resultant glycine would enter the blood at a rate similar to, or possibly rather less than, that of the amino acid, thus giving similar or

${ }^{1}$ Fifty grams of glycine 'not infrequently causes vomiting' (Witts, 1929). 
slightly lower plasma curves. The finding that the peptides gave curves which rose more steeply, attaining higher peaks and suggestive of more rapid absorption, was unexpected. Such differences in the curves might possibly have been attributable to differences in rate of stomach emptying, owing to the doses of peptide having lower osmotic pressures than those of the amino acid. The fact that similar differences were observed in patients with no functional pylorus makes such an explanation most unlikely.

A second possible cause of a greater increase in plasma $\alpha-\mathrm{NH}_{2} \mathrm{~N}$ following the peptides might be that they produced a more powerful stimulus to pancreatic enzyme secretion (via the pancreozymin mechanism) than the free amino acid. The consequent increase in protein entering into the gut with subsequent hydrolysis and reabsorption might contribute significantly to the rise in $\alpha-\mathrm{NH}_{2} \mathrm{~N}$. This explanation seems to be ruled out by the observations that the findings with $\alpha-\mathrm{NH}_{2} \mathrm{~N}$ were approximately paralleled by those using specific glycine nitrogen determinations, and that the chromatographic patterns following glycine and the peptides were identical, showing mainly increases in glycine and alanine. If the increase in $\alpha-\mathrm{NH}_{2} \mathrm{~N}$ following the peptides were contributed to subtantially by absorption of hydrolysed protein from pancreatic secretions, it would be expected that other amino acids would also show a large increase.

A third possible cause of differences in the curves following free glycine and the peptides, differing rates of tissue uptake, also seems unlikely. If peptide had appeared in the blood following oral administration, this possibility would merit serious consideration but the chromatographic findings indicated that only free glycine was present. The possibility that some peptide appeared in the portal blood and in some way (perhaps competitively) retarded liver uptake of glycine cannot, of course, be entirely excluded. However, the shapes of the curves following glycine and the peptides do not suggest that the differences were due to retarded tissue uptake. This would be expected to give a higher peak combined with a more prolonged increase in concentration. It appears most probable that the differences must be due to a faster rate of transfer of glycine into the blood when the peptides are given than when the free amino acid is given.

A hypothetical explanation of this phenomenon may be put forward. The type of relationship between concentration of glycine in the gut lumen and its rate of absorption or transport, as shown in animal experiments (Matthews and Laster, 1965b), and also in man (Fleshler, Butt, and Wismar, 1966), is illustrated in Figure 7. The relationship is not

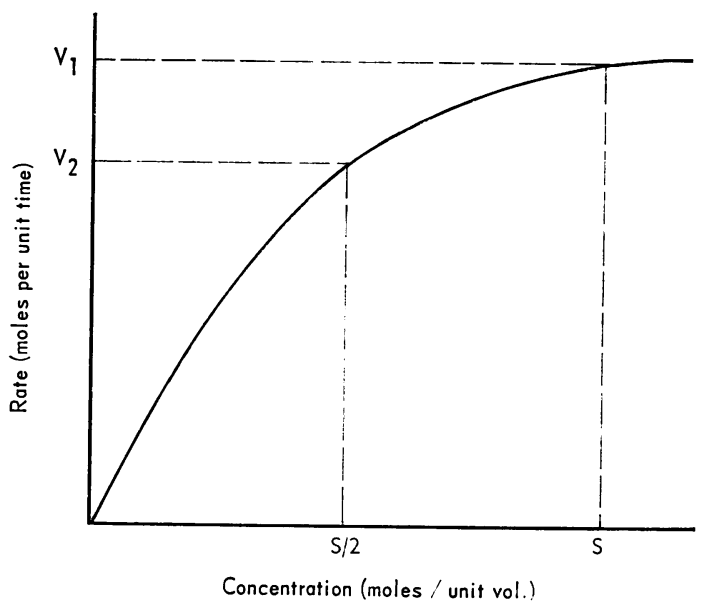

FIG. 7. Curve illustrating relationships between rate of absorption of glycine and its concentration in the intestinal lumen.

linear but conforms to a curve which approaches a maximal rate. If a concentration $\mathbf{S}$ molar is present, the rate of transport is $V_{1}$ moles per unit time. If the concentration is halved, to $\mathrm{S} / 2$ molar, the corresponding rate, $\mathrm{V}_{2}$, is not half $\mathrm{V}_{1}$ but more than half. If it is postulated that glycine and diglycine share a rate-limiting step in transport which precedes intracellular hydrolysis, being handled at this stage in the same way, then the curve relating concentration to rate will be similar for both compounds. Assume that our dose of glycine corresponds to the concentration $\mathrm{S}$ molar. The dose of diglycine we give will then correspond to $S / 2$ molar, and the rate of transport of diglycine, $V_{2}$, will be greater than $V_{1} / 2$. Let us further assume for the sake of argument that the rate of transport of glycine from the gut lumen under our conditions, $V_{1}$, is one mole per unit time. Then the rate of transport of diglycine from the gut lumen under our conditions, $\mathrm{V}_{2}$, will be more than half a mole per unit time. Since half a mole of diglycine contains the same number of glycine units as one mole of glycine, this will mean the transport of more glycine units in a given time when the peptide is given, and, after hydrolysis, will result in the appearance of more free glycine within the cell. This would be expected to lead to more rapid forward transport into the blood. In fact, animal $^{1}$ experiments suggest that such a phenomenon might be demonstrable if it were not for very extensive back diffusion of free amino acid into the gut lumen (Newey and Smyth, 1962). Under our

${ }^{1}$ Experiments in the rat (Matthews, D. M., Craft, I. L., Geddes, D. M., Wise, I. J., and Hyde, C. W., in press (Clin. Sci.), produced similar results to those described here and have confirmed the hypothesis outlined above, showing that the molar concentration/ absorption rate curve is similar for glycine, diglycine, and triglycine. 
more normal conditions, such back diffusion might be avoided. Similar arguments apply to the tripeptide.

It may be noted that if it is accepted that a given quantity of nitrogen enters the blood more rapidly when presented to the gut as peptide than when presented as free amino acid, then, regardless of the precise mechanism of the effect, this demonstrates that hydrolysis within the fluid in the intestinal lumen cannot precede transport. If it did, even instantaneous hydrolysis could not lead to absorption of a given dose of peptide more rapidly than the equivalent dose of free glycine, since after hydrolysis the two doses, each containing the same number of amino acid units, would be indistinguishable. If hydrolysis of the peptide were relatively slow, the concentration of glycine released would be lower than that with free glycine, resulting in slower absorption.

RESULTS IN PATIENTS WITH GASTROINTESTINAL DISEASE The results in patients with no pylorus or a bypassed or non-functional pylorus, eg partial gastrectomy, vagotomy, and pyloroplasty, are clearly compatible with abnormally rapid absorption of glycine and diglycine owing to uncontrolled stomach emptying. Similar results were obtained by Richmond and Girdwood (1962) using casein, and have also been obtained with other substances.

The very severely depressed curves following both glycine and diglycine in three of the cases of idiopathic steatorrhoea suggest gross retardation of absorption in these patients, who also had marked malabsorption of fat and xylose. It is recognized that very many factors affect the shape of a socalled 'tolerance curve' based on oral administration followed by examination of peripheral plasma, and that further investigation is desirable. It would, however, be surprising if impairment of absorption did not play a prominent part in producing the markedly depressed curves we have found. Butterworth et al (1958) concluded that the depressed curves encountered in tropical sprue were not the result of accelerated tissue uptake. which was in fact rather slower than normal, and that renal excretion of glycine was a very minor factor at the $25 \mathrm{~g}$ dose level. Previous workers had also found excretion of glycine to be small (less than $0.5 \mathrm{~g}$ ) during tests of this type (Folin and Berglund, 1922; Gutman and Alexander, 1947) and Richmond and Girdwood (1962) reported that plasma clearance of an amino acid mixture was normal in idiopathic steatorrhoea.

The results obtained with diglycine are not very different from those using free glycine, and do not suggest that deficiency of glycylglycine dipeptidase is a prominent feature of idiopathic steatorrhoea.
No peptide appeared unhydrolysed in the peripheral blood, and comparison of curves after glycine and diglycine suggests that even in the grossly abnormal cases, administration in the form of the peptide still leads to slightly more rapid entry of glycine into the blood, as in normal subjects. Case 14 (Table IV) is exceptional in this respect, the curve following glycine being normal and that following diglycine slightly depressed. However, the general impression is that the use of diglycine is unlikely to have diagnostic advantages in these cases, and that mucosal glycylglycine dipeptidase, even though it may be diminished, is still adequate to deal with the substrate transported.

The impairment of glycine absorption in idiopathic steatorrhoea suggested by the present work is in general agreement with the results of Crane and Neuberger $(1960 \mathrm{a}, \mathrm{b})$ who gave both protein and hydrolysed protein labelled with ${ }^{15} \mathrm{~N}$ and found that absorption was retarded in this condition. It also supports observations (Laster and Matthews, 1963) on the absorption of the non-metabolized amino acid, alpha-aminoisobutyric, which is transported in a rather similar way to glycine. Both our results and those of Crane and Neuberger are difficult to reconcile with those of Richmond and Girdwood (1962) who followed plasma amino acids after giving casein and concluded that absorption was normal. The reasons for these discrepancies remain to be explained. Crane and Neuberger obtained indirect evidence suggesting general deficiency of mucosal peptidases in the coeliac syndrome, and there is some evidence that incompletely hydrolysed protein breakdown products and even whole proteins may enter the blood in this disease (Frazer et al, 1964; Kowlessar, 1967). So far as they go, our results tend to support those of Messer et al (1961), who, using biopsy samples of duodenal mucosa and relating the rate of hydrolysis to total tissue protein, failed to demonstrate retarded hydrolysis of small peptides in the coeliac syndrome.

The results in patients with Crohn's disease and intestinal resections suggest that the small gut must be very extensively affected before absorption of glycine is retarded (Table IV). Normal curves were obtained both in patients with extensive distal resection and extensive proximal resection. In one patient (no. 19) the curve following glycine was severely depressed after resection of the distal small gut, but the general oedema and electrolyte disturbance may have affected transport throughout the intestine. Furthermore, the resection was comparatively recent, and there is some evidence that absorption improves for some months after resections (Althausen et al, 1950). Animal experiments indicate that though the site of maximal transport capacity 
for monoaminomonocarboxylic amino acids is the mid-ileum, as is the site of maximal peptidase activity (Lin and Wilson, 1960; Robinson and Shaw, 1960; Matthews and Laster, 1965a, b), the transport capacity for amino acids is relatively high throughout the small intestine. On the other hand, the site of maximal transport capacity for some monosaccharides appears to be the proximal small intestine (Crane, 1960; Wiseman, 1964). It is tentatively suggested that a proximal resection may retard the absorption of glucose and xylose leaving the ability to absorb free monoaminomonocarboxylic amino acids and possibly small peptides relatively unimpaired. An example of this may be case 6, with an extensive proximal resection, subnormal xylose absorption, a glucose tolerance test compatible with retarded absorption, but normal curves after glycine and diglycine.

The possible nutritional significance of retardation of amino acid absorption in small intestinal disease remains to be assessed.

Only one case of pancreatic malabsorption was investigated in this survey, and gave an abnormally high curve in the glycine test, for reasons that were uncertain. It may have been the result of slow uptake of amino acid by a diseased liver (Folin and Berglund, 1922; Bernhart and Schneider, 1943; Harper and Uyeyama, 1948; Mellinkoff, Jenden, and Frankland, 1954; Richmond and Girdwood, 1962). Amino acid tolerance tests were once used as tests of liver function. Pancreatic disease could, theoretically, lead to impaired tissue uptake of amino acids through lack of insulin. However, this patient was not diabetic. Other work suggests that absorption of glycine and other free amino acids is usually normal in pancreatic disease (eg West et al, 1946; Anfanger and Heavenrich, 1949; Laster and Matthews, 1963), and that the glycine test might be used, like the xylose test, in the differentiation of malabsorption of pancreatic and small-intestinal origin.

Our general conclusions are that the glycine tolerance test, which is rapid and simple to carry out, is not obsolete but may be capable of giving useful clinical information. The diglycine test probably has no diagnostic advantage. The finding, as the result of comparing the two tests, that glycine appears to be absorbed more rapidly when given as the peptide than as the free amino acid, is of considerable physiological interest, and suggests that further investigation along these lines should be undertaken.

\section{SUMMARY}

This paper reports a re-investigation of the 'glycine tolerance test' and an investigation of the absorption of glycylglycine and glycylglycylglycine under similar conditions. Oral doses of free glycine or the peptides were followed by serial estimations of glycine and alpha-amino nitrogen in peripheral plasma.

The results show that when the peptides are given, they appear in the peripheral blood entirely as free glycine. They indicate that a given quantity of glycine is more rapidly absorbed into the blood when administered as a small peptide than as the free amino acid. This is compatible with the view that hydrolysis of the peptides takes place within the cells of the intestinal mucosa, and suggests that a rate-limiting step in transport, common to glycine and the di- and tripeptides of glycine, precedes intracellular hydrolysis.

Patients with gastrectomy, or a non-functional pylorus, apparently absorbed glycine and glycylglycine more rapidly than normal. Three of seven patients with idiopathic steatorrhoea had extremely depressed tolerance curves after glycine and glycylglycine, suggesting severe retardation of absorption. The results did not suggest gross deficiency of glycylglycine dipeptidase in idiopathic steatorrhoea, and the use of glycylglycine did not appear to have any diagnostic advantage in these cases. Results in patients with Crohn's disease and proximal and distal intestinal resections indicated that the small intestine must be very extensively affected before absorption of glycine is obviously retarded.

It is concluded that the glycine tolerance test is capable of giving useful information and that more extensive investigation of tests of absorption of free amino acids and small peptides may contribute further to the knowledge of intestinal absorption in health and disease.

We are grateful to the staff of the Westminster Hospital, to Dr I. A. D. Bouchier, of the Department of Medicine, Royal Free Hospital, and to Dr A. M. Dawson, of Saint Bartholomew's Hospital, for permission to study patients under their care, and to the many volunteers who helped in the investigation. This work was supported by a grant from the Ministry of Health.

\section{REFERENCES}

Alexander, B., Landwehr, G., and Seligman, A. M. (1945). A specific micromethod for the colorimetric determination of glycine in blood and urine. J. biol. Chem., 160, 51-59.

Althausen, T. L., Doig, R. K., Uyeyama, K., and Weiden, S. (1950). Digestion and absorption after massive resection of the small intestine. II. Recovery of the absorptive function as shown by intestinal absorption tests in two patients and a consideration of compensatory mechanisms. Gastroenterology, 16, 126-139.

—, Uyeyama, K., and Simpson, R. G. (1949). Digestion and absorption after massive resection of the small intestine. I. Utilisation of food from a 'natural' versus a 'synthetic' diet and a comparison of intestinal absorption tests with nutritional balance studies in a patient with only $45 \mathrm{~cm}$ of small intestine. Ibid., 12, 795-807. 
Anfanger, H., and Heavenrich, R. M. (1949). Amino acid tolerance tests in children. Amer. J. Dis. Child., 77, 425-436.

Bernhart, F. W., and Schneider, R. W. (1943). A new test of liver function: the tyrosine tolerance test. Amer. J. med. Sci., 205, 636-643.

Butterworth, C. E., Jr, Santini, R., Jr, and Perez-Santiago, E. (1958). The absorption of glycine and its conversion to serine in patients with sprue. J. clin. Invest., 37, 20-27.

Christensen, H. N., Cooper, P. F., Jr, Johnson, R. D., and Lynch, E. L. (1947). Glycine and alanine concentrations of body fluids; experimental modification. J. biol. Chem., 168, 191-196.

- - Streicher, J. A., and Elbinger, R. L. (1948). Effects of feeding individual amino acids upon the distribution of other amino acids between cells and extracellular fluid. Ibid., 172, 515-524.

Crane, C. W. (1961). Some aspects of protein digestion and absorption in health and disease. Postgrad. med. J., 37, 745-754.

- , and Neuberger, A. (1960a). Absorption and elimination of ${ }^{15} \mathrm{~N}$ after administration of isotopically labelled yeast protein and yeast protein hydrolysate to adult patients with coeliac disease. I. Rate of absorption of ${ }^{15} \mathrm{~N}$ yeast protein and yeast protein hydrolysate. Brit. med. J., 2, 815-823.

,$--(1960 b)$. Absorption and elimination of ${ }^{15} \mathrm{~N}$ after administration of isotopically labelled yeast protein and yeast protein hydrolysate to adult patients with coeliac disease. II. Elimination of isotope in the urine and faeces. Ibid., 12, 888-894.

Crane, R. K. (1960). Intestinal absorption of sugars. Physiol. Rev., 40, 789-825.

Drew, R., Dixon, K., and Samuel, E. (1947). Residual defects after sprue: a review of 26 cases. Lancet, 1, 129-134.

Erf, L. A., and Rhoads, C. P. (1940). The glycine tolerance test in sprue and pernicious anemia. J. clin. Invest., 19, 409-421.

Fleshler, B., Butt, J. H., and Wismar, J. D. (1966). Absorption of glycine and L-alanine by the human jejunum. J. clin. Invest., 45, 1433-1441.

Folin, O. (1922). A system of blood analysis. Suppl. III. A new colorimetric method for the determination of the amino acid nitrogen in blood. J. biol. Chem., 51, 377-391.

- , and Berglund, $H$. (1922). The retention and distribution of amino-acids with especial reference to the urea formation. Ibid., 51, 395-418.

Frame, E. G. (1958). The levels of individual free amino acids in the plasma of normal man at various intervals after a high-protein meal. J. clin. Invest., 37, 1710-1723.

Frazer, A. C. (1960). The present state of knowledge on the celiac syndrome. J. Pediat., 57, 262-276.

—, Schneider, R., Morgan, D. B., Sammons, H. G., and Hayward, M. (1964). In The Role of the Gastrointestinal Tract in Protein Metabolism, edited by H. N. Munro. pp. 349-355. Blackwell, Oxford.

Gutman, G. E., and Alexander, B. (1947). Studies in amino acid metabolism. I. Blood glycine and alanine and their relationship to the total amino acids in normal subjects. J. biol. Chem. $168,527-536$.

Hamilton, P. B., and Van Slyke, D. D. (1943). The gasometric determination of free amino acids in blood filtrates by the ninhydrin-carbon dioxide method. Ibid., 150, 231-250.

Harper, H. A., and Uyeyama, K. (1948). Plasma methionine after oral administration of DL-methionine in human subjects. Proc. Soc. exp. Biol. (N.Y.), 68, 296-300.
Heath, C. W., and Fullerton, H. W. (1935). The rate of absorption of iodide and glycine from the gastrointestinal tract in normal persons and in disease conditions. J. clin. Invest., 14, 475-481.

Hier, S. W. (1947). Influence of ingestion of single amino acids on the blood level of free amino acids. J. biol. Chem., 171, 813-820.

Kowlessar, O. D. (1967). Effect of wheat proteins in celiac disease. Gastroenterology, 52, 893-897.

Laster, L., and Matthews, D. M. (1963). Intestinal absorption and malabsorption of the amino acid analogue alpha-aminoisobutyric acid in man. $J$. clin. Invest., 42, 948-949.

Lin, E. C. C., and Wilson, T. H. (1960). Transport of L-tyrosine by the small intestine in vitro. Amer. J. Physiol., 199, 127-130.

McDonald, G. W., Fisher, G. F., and Burnham, V.(1965). Reproducibility of the oral glucose tolerance test. Diabetes, 14, 473-480.

Matthews, D. M., and Laster, L. (1965a). Absorption of protein digestion products: a review, Gut, 6, 411-426.

- - (1965b). Kinetics of intestinal active transport of five neutral amino acids. Amer. J. Physiol., 208, 593-600.

-, Muir, G. G., and Baron, D. N. (1964). Estimation of alphaamino nitrogen in plasma and urine by the colorimetric ninhydrin reaction. J. clin. Path., 17, 150-153.

Mellinkoff, S. M., Jenden, D. J., and Frankland, M. (1954). Postprandial serum amino acid levels in viral hepatitis. Arch. intern. Med., 94, 604-611.

Messer, M., Anderson, C. M., and Townley, R. R. W. (1961). Peptidase activity of biopsies of the duodenal mucosa of children with and without coeliac disease. Clin. chim. Acta, 6, 768-775.

Newey, H., and Smyth, D. H. (1962). Cellular mechanisms in intestinal transfer of amino acids. J. Physiol. (Lond.), 164, 527-551.

Pittman, F. E., and Pollitt, R. J. (1966). Studies of jejunal mucosal digestion of peptic-tryptic digests of wheat protein in coeliac disease. Gut, 7, 368-371.

Richmond, J., and Girdwood, R. H. (1962). Observations on aminoacid absorption. Clin. Sci., 22, 301-314.

Robinson, G. B. (1963). The distribution of peptidases in subcellular fractions from the mucosa of the small intestine of the rat. Biochem. J., 88, 162-168.

- , and Shaw, B. (1960). The hydrolysis of dipeptides by different regions of rat small intestine. Ibid., $77,351-356$.

Saint, E. G., and Weiden, S. (1952). The malabsorption syndrome. Aust. Ann. Med., 1, 58-67.

Schwartz, T. B., Robertson, M. C., and Holmes, L. B. (1955). A micro-diffusion method for the determination of plasma glycine. J. Lab. clin. Med., 46, 657-661.

Smith, E. L. (1960). Peptide bond cleavage. In The Enzymes, edited by P. D. Boyer, H. Lardy, and K. Myrbäck. 2nd ed., vol. 4, pp. 1-10. Academic Press, New York and London.

Smith, I. (1960). Chromatographic and Electrophoretic Techniques, 2nd ed., p. 85. Heinemann, London.

Smyth, D. H. (1964). In The Role of the Gastrointestinal Tract in Protein Metabolism, edited by H. N. Munro, pp. 283-291. Blackwell, Oxford.

West, C. D., Wilson, J. L., and Eyles, R. (1946). Blood amino nitrogen levels. Changes in blood amino nitrogen levels following ingestion of proteins and of a protein hydrolysate in infants with normal and with deficient pancreatic function. Amer. J. Dis. Child., 72, 251-273.

Wiseman, G. (1964). Absorption from the Intestine. Academic Press, New York and London.

Witts, L. (1929). Observations on the metabolism of amino-acids in health and disease. Quart J. Med., 22, 477-491. 